the Proton-synchrotron Division, and apart from minor installations and some road work, there remains only the power house, containing the magnet generators, water-cooling apparatus and electrical sub-station for the whole machine, to be completed. Consideration has already been given to the research programme for the $25-\mathrm{GeV}$. machine, which is planned to become operational in 1960. At an energy of $25 \mathrm{GeV}$. the usual methods of particle analysis and detection break down, and therefore a preliminary period devoted to the study and development of the necessary large and expensive experimental equipment required is desirable. A short list of nine typical experiments which has been drawn up is given in the report.

Unlike the accelerator divisions of the Organization, the Theoretical Study Division is not bound to follow a construction time-schedule, and its scientific output has already been considerable. It will, however, be greatly stimulated when the experimental groups become fully operative, for then it will enjoy the benefit of obtaining its data at source. The Division is mainly concerned with the investigation of the so-called elementary particles, their number, structure, properties and interactions. The lists of publications originating from the Division, and the colloquia held at the Institut de Physique in Geneva until June 19, 1957, and at the Meyrin site until the end of the year, together with the lectures and colloquia of the Division at Copenhagen, are given in appendixes to the report. The number of members of the Division greatly increased during the year and included senior physicists from non-member countries who were able to pursue their studies in the Organization's laboratories through grants provided by the Ford Foundation.

In the Scientific and Technical Services Division, the main spheres of development of experimental techniques were liquid-hydrogen bubble chambers and electronic evaluation of track photographs. In addition, a health physics section was started, and the Division continued to administer the Scientific Information Service and the Cosmic Ray Research Group.

The hydrogen-liquefying plant mentioned in last year's report was installed in the summer of 1957 and liquid hydrogen was produced for the first time in July 1957. The plant produces about 32 litres per hour. The $10-\mathrm{cm}$. bubble chamber was completed during the year, and an illustration of it, together with photographs of other equipment, is included in the annual report.

\title{
THE AUTOMATIC COMPUTING ENGINE AT THE NATIONAL PHYSICAL LABORATORY
}

$\mathrm{T}$ HE Automatic Computing Engine, or ACE, is the latest digital computing machine to be built at the National Physical Laboratory for the use of its Mathematics Division. This new machine, with its increased speed of operation, its larger storage capacity and its many additional functional facilities, is a considerable advance as a computing tool on its predecessors, the experimental pilot-model, ACE, and its engineered counterpart, DEUCE.

It is not easy to assess the speed of a machine from the times taken to carry out the elementary arithmetical and logical operations. This is particularly true of any machine with a high-speed store of less than about 8,000 words, on which many problems will demand the use of the backing-up store. The times taken for a few basic computations probably give as accurate a picture of the overall speed as can be obtained without a detailed study.

(1) The zeros of a polynomial of the 16th degree may be obtained in an average time of 15 sec. Because of the long word-length (48 digits), accurate roots even of very ill-conditioned polynomials of this degree will be obtained. Polynonials of degrees up to about 250 may be found without using the drumstore, though no particular loss in speed results from using the drum on such problems.

(2) A set of simultaneous equations of order 30 can be solved in about 5 sec. Even if none of the coefficients is zoro, sets of orders up to 170 may be solved, the time taken varying approximately as the eube of the order but being slightly less for higher orders.

(3) The solution of Poisson's equation, $\nabla^{2} V=4 \pi p$ on a square with 400 mesh points, may be obtained in about 75 sec. This time is for the direct solution of the finite difference equations and will give values correct to at least ten decimals. After solving one such problem, solutions corresponding to different distributions of $\rho$ may be obtained in about 15-20 sec. each.

ACE is a serial computer in which numbers and instructions have 48 binary digits; the digit-rate is 1.5 million per second, and therefore its word-time or minor cycle is $\mathbf{3 2}$ microseconds.

The main working store consists of twenty-four mercury delay lines each containing thirty-two words, circulating in a major cycle of 1,024 microseconds. Rapid-access storage is obtained by using mercury delay lines with capacities of one, two and four words. The backing store consists of four magnetic drums containing a total of 32,768 words.

The ACE instruction specifies a three-address operation of the form ' $A$ function $B$ to $D$ ', where $A$ and $B$ are the store-addresses of the operands and $D$ is the store-address to which the result of the operation is to be sent. Instructions also specify a further store-address, $N$, from which the next instruction is to be extracted. The time needed to execute this operation (for single-length numbers) is 32 microseconds, and since each operation may be followed immediately by another, a maximum rate of operation of 30,000 per second is possible.

The drum-store, the multiplier and the divider are independent units. These are put into operation by the appropriate instructions and then work inde. pendently until they have completed their operation. This feature provides a parallelism of operation, since a multiplication, division, drum-transfer and a series of ordinary operations could be in progress at the same time.

The three-address operation of the machine allows the multipliers and multiplicand to be selected and the multiplication process to be initiated by a single 
instruction. The product is formed in fourteen minor cycles, that is to say, in 430 microseconds. The division process is similarly specified, and the quotient (rounded or unrounded) is formed in approximately 1.5 milliseconds. The divider also contains an automatic standardizing process for use in floating-point arithmetical operations.

The magnetic drum-store has a total capacity of 1,024 long delay lines, that is to say, about 1.5 million digits. There are four drums each having 256 tracks, each track storing the contents of one delay line. Each drum has sixteon read heads and sixteen write heads, the 256 tracks being obtained by moving the heads as one unit into one of the sixteen discrete positions. Each drum is 6.75 in. long by 5 in. in diameter, allowing a linear digit packing of 100 per in. The drum is driven by a hysteresis motor running synchronously at 12,000 r.p.m. and is phasecorrected so as to rotate exactly once in five major cycles of the machine.
Punched card and magnetic tape equipment will be provided for input and output. The installation will initially consist of two machines: (1) a broadside card reader, running at 450 cards per min.; (2) a broadside card punch running at 100 cards per min. In order to read or punch 80 columns of a card the computer has an 80-digit input dynamicizer and an 80 -digit output staticizer.

The ACE has been designed and constructed to allow easy maintenance. Extensive marginal checking facilities are provided and the chassis units are designed to give complete accessibility to all components, valve connexions, etc.

The machine is housed in ten cabinets, each having a cooled air-circulation system. Each cabinet is fitted with a rising door which permits immediate access to all the twenty-four chassis units contained therein. The number of valve envelopes is about 6,000 .

J. H. WILKINSON

D. W. Davres

\section{THE ADÉLIE PENGUIN}

$\mathrm{D}^{\mathrm{t}}$ ETAILED observations of the Adélie penguin, Pygoscelis adeliae, have been carried out by Dr. W. J. L. Sladen of the Falkland Islands Dependencies Scientific Bureau and the Edward Grey Institute, University of Oxford*.

The Adélie is antarctic and circumpolar in its distribution, but does not breed north of the South Sandwich Islands and Bouvet Island. It is the most abundant and widely distributed of all the antarctic penguins.

Two rookeries were studied between 1948 and 1951, one at Hope Bay, Graham Land, and the other at Signy Island, South Orkneys. At both of these there survived a small number of birds that had been ringed two seasons before. The only certain way of determining the sex of these penguins, other than by dissection, is to mark breeding birds before egg-laying and to continue observing them during the early part of the breeding season. Casuals and non-breeders cannot be sexed unless marked and recovered breeding in another season.

The diversity in behaviour of a penguin community appears to be due to three factors which are often inter-related: variation among individuals, differences in 'intensity' of behaviour, and differences in age and breeding experience. Emphasis is laid on the differences in age and breeding experience, and some evidence is given from field observations and dissections to show that this is an important factor.

Suggested age-groups divide a population seen at the rookery into five categories: the experienced (established) breeders, the inexperienced (unestablished) breeders, the non-breeders ('wanderers') in adult plumage, the non-breeders in immature plumage (yearlings), and the nestlings.

The established breeders, probably four or five years old and more, come back to their former nestsites independently of their former mates. Such a bird may 'keep company' with another bird of the opposite sex until its true mate returns.

* Falkland Islands Dependencies Survey. Scientific Rcports, No. 17 The Pygoscelid Penguing. 1: Methods of Study. 2: The Adéli Penguin Pygoscelis adeliae (Hombron and Jacquinot). By Dr. William J. L. Sladen. Pp. $1+97+12$ plates. (London: H.M. Stationery Office, 1958. Published for the Colonial Office.) 478. 6 d. net.
The unestablished breeders have furst to establish themselves and pair off. Their behaviour differs somewhat from that of the established birds, though with some overlap due to individual variations. They often arrive back later than the established breeders, and take up territories on the periphery of the colonies, or fill vacant places within them. They are the cause of most of the fighting. Courtship behaviour is more prolonged, egg and chick mortality higher and breeding efficiency lower.

The non-breeding 'wanderers' in adult plumage are two or three years old and can be seen wandering around the rookery in small parties, and even visiting rocky outcrops up to $1,000 \mathrm{ft}$. above sea-level far away from the breeding area. Some of them build poor nests, or occupy vacant sites for a short time before moving elsewhere or being turned out by older birds. Of the wanderers which were dissected, some showed undeveloped gonads despite their adult plumage. It is this age-group which, together with some of the unestablished birds, under favourable conditions, occupy new ground and thus extend the range of the species. They will also quickly take the opportunity to fill any gap in the colonies, and may during the re-occupation period occupy territory in anticipation of the following season.

The yearlings, which are easily recognized by their white throats, normally live among the pack ice, but are occasionally seen at the rookery, where they associate with the wanderers. Several were seen building nests in a half-hearted way on the edge of colonies; but, like the wanderers, they never stayed in one place for long.

Describing the displays and postures of the Adélie penguin, Sladen shows that during the first and second fasting periods the ecstatic display is confined to the male, its most important function appearing to be in pair-formation. A lone male by this display can attract a nearby female to its nest. After the eggs have hatched (or should have hatched), it is also seen in females, though on a diminished scale. It is more commonly seen among the younger and unestablished breeders or non-breeders than among the established breeders. 